\title{
AUTOLOGOUS STEM CELL TRANSPLANTATION AND IMMUNOMODULATION IN AMYOTROPHIC LATERAL SCLEROSIS
}

\author{
David Uriel Ayllón-Álvarez ${ }^{1}$, Mariana Neri-Calixto², César Humberto Aparicio-Albarrán ${ }^{3}$, Hugo Mendieta Zerón ${ }^{4}$ \\ Correspondence:drmendietaz@yahoo.com \\ ${ }^{1}$ Faculty of Medicine, Autonomous University of the State of Mexico, Toluca, Mexico State, Mexico \\ ${ }^{2}$ Faculty of Medicine, Autonomous University of the State of Mexico, Toluca, Mexico State, Mexico \\ ${ }^{3}$ ISSSTE Hospital General Toluca, Mexico. \\ ${ }^{4}$ Faculty of Medicine, Autonomous University of the State of Mexico and Mónica Pretelini Sáenz, Maternal-Perinatal Hospital, Toluca, \\ Mexico State, Mexico
}

\section{Article History:}

Received: June 1, 2021

Accepted: June 30, 2021

Published: July 1, 2021

\section{Cite this as:}

Ayllón-Álvarez DU, Neri-Calixto

$M$, Aparicio-Albarrán $\mathrm{CH}$,

Mendieta Zerón H. Autologous

stem cell transplantation and immunomodulation in

amyotrophic lateral sclerosis.

Malang Neurology Journal;

2021.7: 140-145.

http://dx.doi.org/10.21776/ub.mnj .2021 .007 .02 .12

\section{ABSTRACT}

Background: Amyotrophic lateral sclerosis (ALS) is a rare chronic degenerative disease. The only approved treatment, Riluzole, is not available and is not economically affordable for most patients. Objective: To report here a case of a pregnant woman 27 years of age with ALS, confirmed through electromyography. After spontaneous abortion and 88 days without clinical improvement and dependent on mechanical ventilation, treatment with autologous stem cell transplantation and immunomodulation was decided upon for the patient.

Methods: The patient received a daily subcutaneous (s.c.) dose of $300 \mu \mathrm{g}$ human Granulocyte-Colony stimulating factor for a period of 3 days. Peripheral blood mononuclear cells were isolated by leukapheresis. These cells were self-transplanted to the patient $5 \mathrm{cc}$ via lumbar puncture and $5 \mathrm{cc}$ intravenously (i.v.). The immunomodulation treatment was based on the surface-fixation method.

Results: The patient remained hospitalized for an additional 249 days, at which time when she was contrareferred to a secondary-level health institution.

Conclusion: A treatment scheme combining ASCT (Autologous Stem Cell Transplantation) and immunomodulation, based on the surface-fixation method, could be an option to get a large period of no progression stage in ALS.

Keywords: Amyotrophic lateral sclerosis, autologous stem cell transplantation, puerperium, surfacefixation method.

\section{Introduction}

The Amyotrophic lateral sclerosis (ALS) incidence is of 23.9 cases per 100,000 people annually. ${ }^{1,2}$ The main characteristic of this disease is a selective death of motor neurons in the brainstem, motor cortex and spinal cord. A high genetic and phenotypic variability is inherent to this neurodegenerative disorder. Unfortunately, in most cases the cause cannot be determined. There are two presentations: 1) Sporadic ALS (SALS) ( 90\%), and 2) familial ALS (FALS). ${ }^{3}$ According to El Escorial Criteria (EEC), ${ }^{4,5}$ the criteria are grouped into categories A and B and the firsts are needed for the diagnosis of ALS, with the absence of the seconds (Table 1). ${ }^{6}$

Currently, the cure for ALS is far from being achieved in the near future. The only approved treatment is Riluzole, registered in 1995 by the U.S. Food and Drug Administration (FDA), exerting a moderate effect on the disease process, increasing the patient's life span by $2-3$ months on average. ${ }^{7,8}$

Stem cell therapy, included in recent years as an alternative to restore the correct cellular function in several diseases, also adds the possibility of using this technique in neurodegenerative disorders. ${ }^{9,10}$ This procedure aimed to get the cell replacement keeping on the functions, searches for other options of recovery, such as, the extracellular motor neuronal environment modification by means of a trophic and neuroprotective effect. ${ }^{11}$

To suppress the neurotoxic state and at the same time retain and promote protective functions, immunotherapies could represent a new strategy to treat ALS. Specifically, increasing regulatory T-lymphocyte levels in patients with ALS in early stages of the natural history of the disease could be of therapeutic utility. ${ }^{12}$ In line with the earlier information, in the 1950s, Ruiz-Castañeda developed the surface-fixation method, demonstrating the antigenantibody union. ${ }^{13}$ This method was then employed to produce a personalized immunogenic component in a diverse range of diseases. ${ }^{14-16}$

\section{Antigen surface fixation method}

Briefly, the test is performed on the surface of filter paper and is submitted to the washing effect of a stream of isotonic $\mathrm{NaCl}$ solution. While normal serum is washed together with the antigen, the immune serum produces the 
antigen fixation to the paper at the site it was deposited. This test was first performed on a slide. A $3 \mathrm{~mm}$ wire loop of antigen is mixed with the whole blood taken with a 2 $\mathrm{mm}$ loop. The mixture is rotated in to promote the agglutinated antigen margination. In about 1 or $2 \mathrm{~min}$, a thick blue ring is formed and surrounds the red cells which have separated from the antigen. In negative reactions, the mixture remains a uniform greenish color often surrounded by a reddish ring formed by marginated cells. ${ }^{13}$

The antigen surface method for a urine sample can be performed as follows: In a bottle of $1 \mathrm{~L}, 250 \mathrm{~mL}$ of $96^{\circ}$ ethanol is added, then the first morning urine of three consecutive days is collected there..$^{17}$ (Figure 1).

From the treated urine a precipitate is obtained, which after 24 to 48 hours can be concentrated by centrifugation. The pellet is treated with a volume of between 5 and $10 \mathrm{~mL}$ of saline solution, and after three dilutions one obtains the final concentration to impregnate the paper. The filter paper is treated with bromophenol for one minute, acid alcohol and water. ${ }^{18}$ The dye is a $0.2 \%$ solution of bromophenol, and the fixative solution has $50 \%$ ethyl alcohol and $10 \%$ acetic acid. At least two strips of filter paper dried on the stove can be impregnated with the problem urine. From a volume of $500 \mathrm{~mL}$ of urine, 1 or 2 grams of wet precipitate can be obtained.

\section{Immunodulation}

The Ruiz Castañeda treatment, is based on the immunological and inflammatory response modulation through autologous antigens (own antigens). After the urine antigen surface method, the antigen is titrated by surface binding tests using the patient's serum until a satisfactory solution is obtained which is filtered through Millipore paper and diluted in a proportion of 2 to $5 \%$ in a saline solution, containing as a preservative $0.5 \%$ phenol. The solutions are distributed in sterile vials for storage at $4{ }^{\circ} \mathrm{C}$. $500 \mathrm{ml}$ of urine yields an average of $200 \mathrm{ml}$ of antigenic material properly diluted, titrated and sterilized. In the alcoholic supernatant another part of the antigen can be recovered by evaporating it, obtaining 5 to $10 \mathrm{~mL}$ of antigen, useful, if required, for serological diagnosis. ${ }^{19}$ Once isolated, the antigenic solution is administered s.c. in the patient's arm 2 days/weekly, increasing the dose to 10 cc per week until the final dose of $100 \mathrm{cc}$. This treatment can be kept for 3 years but depends on the clinical response. The self-antigens isolation with this method has been useful to design vacuums to mitigate the immune reaction (desensitization or hypo-sensitization), offering another alternative to modify the immune system's response. The principal aim of this article is to report the experience of one ALS case treated with ASCT and immunomodulation with the surface-fixation method. This clinical approach was approved by the Ethics in Research Committee of the "Mónica Pretelini Sáenz" Maternal-Perinatal Hospital (HMPMPS), Health Institute of the State of Mexico (ISEM), Toluca, Mexico.

\section{Case Report}

The Pregnant female, aged 27 years, 14 weeks of gestation. Referred from the primary-care facility due to respiratory compromise. Main data: native of the State of Mexico,
Mexico, evangelist, primary school, homemaker. Smoking, alcoholism, or other addictions were denied. Menarche at 15 years, five pregnancies, three vaginal deliveries, one abortion.

Table 1. The Escorial Criteria for Amyotrophic Lateral Sclerosis

A-criteria:

A1: Neuropathological, electrophysiological or clinical examination that confirms degeneration of the lower motor neuron

A2: Degeneration of the upper motor neuron found on clinical inspection

A3: Progressive damage beyond typical nerve- supply areas.

B-criteria:

B1: Electrophysiological or neuropathological findings typical for other diseases that could explain the degeneration of the upper and lower motor neuron

B2: Findings in imaging studies that can explain the clinical symptoms. Diagnosis of ALS requires the taking of an extensive patient history and a thorough clinical and electrophysiological examination. The electrophysiological tests can demonstrate the involvement of the lower motor neuron in affected, and frequently also in clinically not affected, body regions. The electromyography includes the next four body regions to be evaluated: brain stem (bulbar), cervical (neck and upper limbs), thoracic (trunk, abdominal wall) and lumbosacral (lumbar spine and lower limbs).

Modified from: Brooks BR, et al. (5). Updated criteria considering the patient's Electromyography (EMG) results (Table 2).

Table 2. Awaji-Shima Consensus Conference Recommendations for The Application Of Electrophysiological Tests for The Diagnosis of Amyotrophic Lateral Sclerosis

1. Principles (from Airlie House Criteria)

A) The Amyotrophic Lateral Sclerosis (ALS) diagnosis requires the following:

1) The presence of Neuropathological, electrophysiological or clinical data confirming Lower Motor Neuron (LMN) function worsening

2) In the physical examination clear information of Upper Motor Neuron (UMN) affection

3) Symptoms or signs with a progressive trend within a single region or more than one, as proved by electrophysiological tests, clinical history or physical exploration.

B) The absence of

1) other disease processes that might explain the LMN and/or UMN degeneration signs, through electrophysiological or pathological evidence.

2) evidence through Magnetic Resonance Image (MRI) of other diseases that could be the cause of the clinical and electrophysiological alterations found.

\section{Diagnostic categories}

- Definite ALS: positive LMN and UMN signs confirmed by clinical or electrophysiological studies. The bulbar region and two or more spinal regions must be affected (or three spinal regions).

- Probable ALS: LMN and UMN signs (through clinical or electrophysiological examination) in at least two regions but always taking into account that some UMN signs must be rostral to LMN signs.

- Possible ALS: LMN and UMN signs in only one region; or when in two or more regions the only signs founds are UMN; or when LMN signs are found rostral to those of UMN. Lack of neuroimaging and clinical laboratory studies and other diagnoses have been excluded.

Modified from Finsterer J, Stöllberger C. Apply Awaji-shima Consensus Conference Criteria Before Diagnosing Amyotrophic Lateral Sclerosis. Open Neurol J. 2013;7:4-6

Present disease: One year before the hospitalization, the patient referred dorsal pain, and 6 months before hospitalization she experienced fatigue easily and dyspnea on medium exertion, progressing to exacerbation at rest. At the patient's first medical consultation, the diagnosis of pulmonary fibrosis was considered. She arrived at the HMPMPS with mechanical ventilation. 


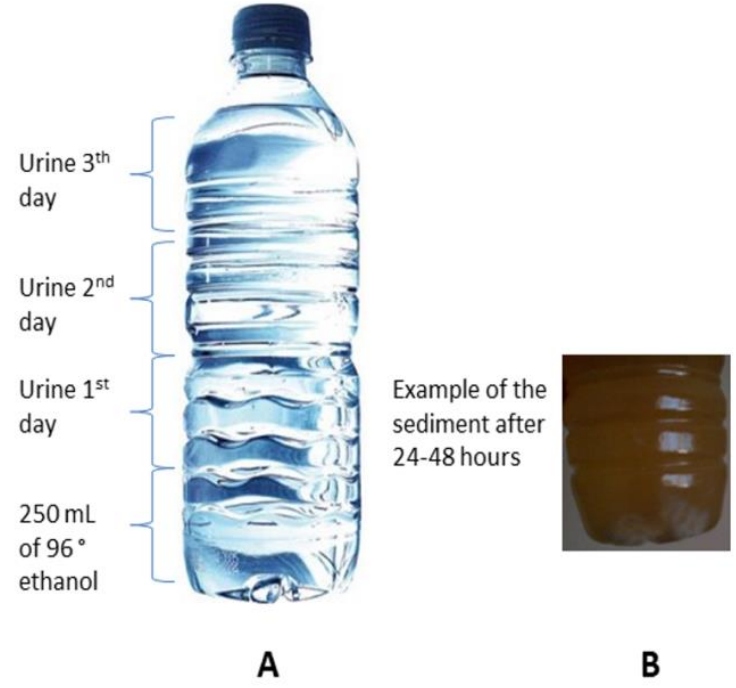

Figure 1. Urine recollection process and sediment formation

On physical examination, the patient had 110/71 mmHg of blood pressure; respiratory rate: 32 breaths per minute, cardiac rate: 78 beats per minute, mean blood pressure: 84 $\mathrm{mmHg}$ and body temperature: $36.5^{\circ} \mathrm{C}$. On general inspection, the patient exhibited cachexia, with pale skin and tegument + , with good hydration, accepting oral diet, and with optimal uresis and evacuations. Neurological evaluation showed Glasgow 11 (eyes $=4$, verbal $=5$ previous to the intubation, motor $=2$ ), isochoric and normal reflective pupils, preserved mental status as well as the cranial nerves although the glossopharyngeal and vagus were not evaluated due to the orotracheal tube, decreased distal muscular strength $2 / 5$, mainly in the right-pelvic and the right-limb, tendon reflexes abolished the sensitivity preserved. Cardiovascular: Carotid pulse with normal tone and intensity, rhythmic heart sounds, with good tone and intensity without aggregated phenomena; hemodynamically stable. Respiratory: In mechanical ventilation support, fraction of inspired oxygen ( $\mathrm{FiO} 2)$ : $50 \%$, bilateral basal lung fields with hypoventilation, and saturation of peripheral oxygen (SPO2): $99 \%$ (pulse oximetry). At baseline, the pulmonary function test demonstrated median Forced Vital Capacity (FVC) of 55.5\% (25th and 75th quartiles $=39.5$ and 74 , respectively). Digestive system: accepting oral diet; soft abdomen, painless on palpation, peristalsis present, slightly increased. Hepatometabolic: capillary glucose $92 \mathrm{mg}$ and $89 \mathrm{mg}$ in venous sample; total proteins: $7.4 \mathrm{~g} / \mathrm{dl}$, and albumin: $4.4 \mathrm{~g} / \mathrm{dl}$. Obstetrics and Gynecology: external genitalia, unaltered, no hematic losses and good uterine involution. Renal: urine volume of $3.2 \mathrm{ml} / \mathrm{kg} / \mathrm{h}$. The coagulation, hepatic and hematic profiles were within normal ranges. The lumbar puncture showed the next data: Na: $146 \mathrm{mmol} / \mathrm{L}$ (reference range: 136-145), $\mathrm{K}: 2.5 \mathrm{mmol} / \mathrm{L}$ (reference range: 3.5-5.1), $\mathrm{Cl}: 124 \mathrm{mmol} / \mathrm{L}$ (reference range: 98-107), lactate dehydrogenase (LDH): 21 U/L (reference range: 100-190), color: water of rock, appearance: clear, density: 1.008 , cellularity: 4 cells $/ \mathrm{mm} 3$, glucose: $73 \mathrm{mg} / \mathrm{dL}$, proteins: $26 \mathrm{mg} / \mathrm{dL}$. In the paraclinical studies the electrocardiogram was normal, the Magnetic Resonance Image (MRI) was negative for any type of lesion and the Electromyography (EMG) demonstrated the affected neuron system (Figures $2 \mathrm{~A}$ and $2 \mathrm{~B}$ ). At the time of hospitalization, the patient had a score of 9 in the ALS Functional Rating Scale (ALSFRS) [0= worst and $40=$ best] and of 12 in the ALS Functional Rating Scale Revised (ALSFRS-R) $[0=$ worst, $48=$ best $]$.

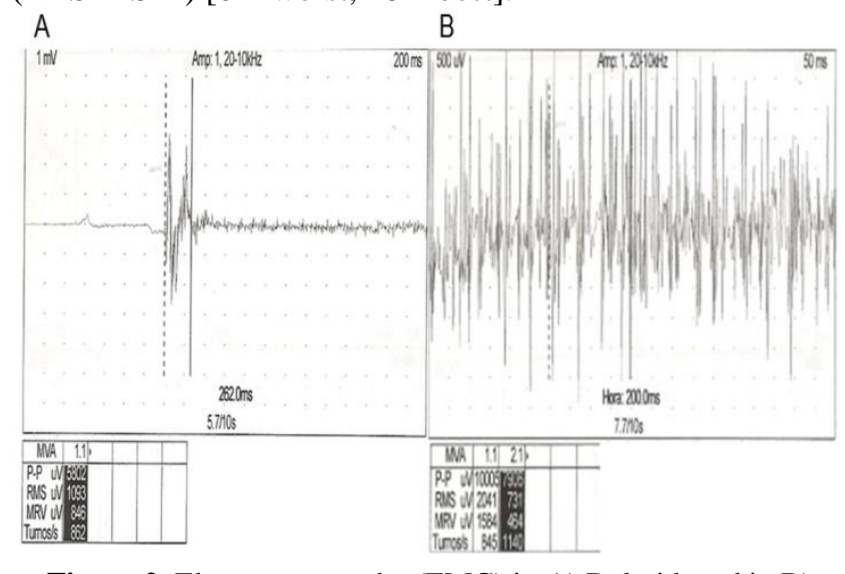

Figure 2. Electromyography (EMG) in A) Deltoid, and in B) Brachiis. The test shows fibrillation potentials.

\section{Treatment}

Previously, during the patient's hospitalization, she developed spontaneous abortion, and it was necessary to do the tracheostomy. After 88 days of hospitalization with an unfavourable outcome, without the possibility of acquiring Riluzole and with the patient's dependence on mechanical ventilation, treatment with Autologous Stem Cell Transplantation (ASCT) and immunomodulation was decided upon.

\section{Autologous cell transplant}

The patient received human Granulocyte-Colony Stimulating Factor (G-CSF) (Filgrastim) (Neupogen, Basel, Switzerland) in a daily subcutaneous (s.c.) dose of $300 \mu \mathrm{g}$ for three days. Peripheral blood mononuclear cells (PBMC) were isolated by leukapheresis (Amicus ${ }^{\mathrm{TM}}$-Separator, Fenwal Inc.). This procedure lasted for $1 \mathrm{~h}$. These cells were self-transplanted to the patient, $5 \mathrm{cc}$ via lumbar puncture and $5 \mathrm{cc}$ intravenously (i.v.).

\section{Immunomodulation therapy}

Following the surface-fixation method, the self-antigen isolation and preparation was developed at the "Dr. Maximiliano Ruiz Castañeda" Instituto de Alergias y Autoinmunidad (IAAMRC), Acambay, State of Mexico, Mexico. On January 27, 2012, the formula administration began until the final dose of $100 \mathrm{cc}$.

\section{Clinical evolution}

Two weeks after ASCT, our patient exhibited fever, suppressed successfully with metamizol and morphine i.v. After achieving clinical stabilization, she was transferred to the Maternal-Fetal Service, where she remained for 249 days due to her dependency on mechanical ventilation. The patient was finally contrareferred to a secondary-level health hospital while continuing to be dependent on mechanical ventilation.

\section{Discussion}

In ALS, unfortunately, the leading cause of death is respiratory failure due to progressive paralysis of the diaphragm, and this fatal event occurs within 3-5 years of the patient's initial symptoms. The neurons death of the anterior horn and the loss of motor axons explain the 
typical amplitude reduction in the muscle action potentials. $^{20}$ In the case of our patient, she was already dependent on mechanical ventilation when she arrived at our Hospital.

On the other hand, the EMG is a useful tool for diagnosing ALS and excluding other pathologies, such as neuromuscular disorders, other motor neuron diseases and peripheral neuropathies. ${ }^{1}$ In the case of our patient, there was no doubt about the diagnosis of ALS.

Despite extensive world research on cell transplantation. ${ }^{21,22}$ there are some topics that must be solved, for example, the cell type selection that would guarantee the highest success rate for a transplant and with important neurogenic properties. Equally important, is choosing the technique, combined with the cell type capable of coping with the unfavourable microenvironment and at the same time with the ability to get the neural-tissue restoration.

The ASCT therapeutic utility mixed with surface-fixation method aimed to develop a personalized immunomodulation was focused on two targets. First, to prevent disability progression, ${ }^{23}$ and second, to get an immune desensitization with the knowledge of ALS to have a sustained unregulated immune response. ${ }^{24,25}$ Among the issues to take into account in the field of cell transplant are 1) Neuronal electrophysiology; 2) synapse formation and 3) synergy with the host tissue of the best cell type that can induce the desired regeneration in the safest and most efficient way. $^{26}$ Axonal regeneration and neurons replacement is the expected challenge to reach thanks to the neurotrophic factors. ${ }^{27}$

In exploring clinical trials using Mesenchymal Stem Cells (MSC), several groups worldwide have achieved promising and interesting results, while, in another approach, it has been tried to enhance the stem cells action to avoid neuronal degeneration (neuroprotection theory), rather than looking for the replacement of lost neurons.

A Phase I trial was published in 2012 by Riley and colleagues in which neural fetal cells were implanted in 12 patients with ALS at the lumbar level. The group reported no adverse events with the transplants, findings that are in line with the already confirmed safety of this technique. ${ }^{28}$ Likewise, other groups have made research with the human fetal Spinal Cord-Derived (SCD) neural stem-cell line biological NSI-566RSC, with proved efficacy and safety based on preclinical studies with small-animal models of ALS. ${ }^{29,30}$

Having a general visualization of the available information, it is appreciated that the commented techniques and others like them have shown encouraging results in animals, but still limited in humans due to the scarcity of studies carried out to date.

It has been postulated that autologous stem cells, through complex and precise induction mechanisms, can be successfully differentiated into mature neurons with all their functions, for the purpose of repopulating damaged areas. Actually, peripheral blood CD133 stem cells by a series of complex and specific steps can be differentiated into neurons. ${ }^{31}$ Notwithstanding, the neuroprotective method has not been elucidated, and it is unclear whether an efficient development of functional neurons contributes for this rescue. On the other hand, it seems quite plausible to think that CD133 cells neuroprotect by means of by as yet unknown cellular mechanisms or by already identified growth factors.

The group of Martínez et al. had attempted stem cell transplantation for ALS in women and men ${ }^{32}$ demonstrating that autologous CD133 stem cell transplantation into the frontal motor cortex is a safe and well-tolerated procedure in patients with ALS. To our knowledge, our report comprises the first case of ASCT in the State of Mexico (population, 16 million people).

Due to several issues, some researchers lack confidence regarding the benefits of ASCT in patients suffering from ALS. One of the main concerns is about the mutations emergence possibility in some of the most susceptible genes such as C9ORF72, FUS/TLS, SOD1, TDP-43, $\mathrm{UBQLN} 2$ and $\mathrm{VCP} / \mathrm{p} 97 .{ }^{33}$ Whether this is true remains a matter of debate.

A controversy exists about how to decide the celltransplantation dose and route in neurological diseases. In cases of heterologous transplant, the most usual dose employed is expressed in $\mu 1 .{ }^{34}$ In this initial approach, considering a lack of possible rejection, $5 \mathrm{cc}$ of leukapheresis were injected without immediate collateral effects, but with fever 2 weeks after the procedure, an adverse event not registered by Martínez et al. ${ }^{32}$

Despite the fact of the long hospital stay of our patient, it cannot be attributed to the ASCT, as the reported survival time after this procedure is even longer, but probably it was documented a plateau in the state of disease progression thanks to the immunomodulation treatment. In fact, the survival of the patient of this report was longer than that reported in the casuistry of Mayaux et al. with 90 patients affected by ALS with a mortality after 3 months and one year of $46 \%$ and $71 \%$ respectively. ${ }^{35}$ By contrast, the group of Vianello et al. reported outstanding results after tracheostomy in patients with ALS (median survival after tracheostomy of 21 months). ${ }^{36}$ It is well known that survival after tracheostomy in ALS is generally increased, with the stronger effect in patients younger than $60^{37}$ but due to the lack of novel pharmacological options it is mandatory to look for more alternatives.

A limitation of the tested approach is that it was not perform a deeper study through the "omics" sciences to characterize more precisely the isolated antibody-antigen component in the filter paper. This is a process pending to contribute in the analysis of the hypothetical tolerogenic effect of the Ruiz-Castañeda method.

ALS is a tremendous challenge for medical science so more research focused on rehabilitation and experimental techniques of regenerative medicine is justified and pending. ${ }^{38,39}$

\section{Conclusion}

A treatment scheme combining ASCT and immunomodulation, based on the surface-fixation method, could be an option to get a large period of no progression stage in ALS. 


\section{Acknowledgement}

Authors thank Dr. Adrián Gerardo Vergara-Cuadros of the "Mónica Pretelini Sáenz" Maternal-Perinatal Hospital (HMPMPS) for his excellent help with the leukapheresis procedure and Maggie Brunner M.A., for the English language style correction.

\section{Conflict of Interest}

The authors declare that there is no conflict of interst.

\section{References}

1. Sorenson EJ. The electrophysiology of the motor neuron diseases Neurol Clin; 2012. 30(12):605-620. DOI: 10.1016/j.ncl.2011.12.006

2. Mehta P, Kaye W, Bryan L, Larson T, Copeland T, $\mathrm{Wu} \mathrm{J}$, et al. Prevalence of amyotrophic lateral sclerosis - United States 2012-2013. Morb Mortal Wkly Rep Surveill Summ Wash DC 2002; 2016. 65(8):1-12. DOI: 10.15585/mmwr.ss6508a1

3. Poppe L, Rué L, Robberecht W, Van Den Bosch L. Translating biological findings into new treatment strategies for Amyotrophic Lateral Sclerosis (ALS). Exp Neurol; 2014. 262 Pt B:138-151.

DOI: 10.1016/j.expneurol.2014.07.001

4. Brooks BR. El Escorial World Federation of Neurology criteria for the diagnosis of amyotrophic lateral sclerosis. Subcommittee on Motor Neuron Diseases/Amyotrophic Lateral Sclerosis of the World Federation of Neurology Research Group on Neuromuscular Diseases and the El Escorial «Clinical limits of amyotrophic lateral sclerosis» workshop contributors. J Neurol Sci; 1994. 124 Suppl:96-107. DOI: 10.1016/0022-510x(94)90191-0

5. Brooks BR, Miller RG, Swash M, Munsat TL, World Federation of Neurology Research Group on Motor Neuron Diseases. El Escorial revisited: Revised criteria for the diagnosis of amyotrophic lateral sclerosis. Amyotroph Lateral Scler Mot Neuron Disord; 2000. 1(5):293-299.

DOI: $10.1080 / 146608200300079536$

6. de Carvalho M, Dengler R, Eisen A, England JD, Kaji $\mathrm{R}$, Kimura $\mathrm{J}$, et al. Electrodiagnostic criteria for diagnosis of ALS. Clin Neurophysiol; 2008. 119(3):497-503. DOI: 10.1016/j.clinph.2007.09.143

7. Paillisse C, Lacomblez L, Dib M, Bensimon G, Garcia-Acosta S, Meininger V. Prognostic factors for survival in amyotrophic lateral sclerosis patients treated with riluzole. Amyotroph Lateral Scler Mot Neuron Disord; 2005. 6(1):37-44. DOI: $10.1080 / 14660820510027035$

8. Miller RG, Mitchell JD, Moore DH. Riluzole for amyotrophic lateral sclerosis (ALS)/motor neuron disease (MND). Cochrane Database Syst Rev; 2012. (3):CD001447. DOI: 10.1002/14651858.CD001447

9. Li M, Guo K, Ikehara S. Stem cell treatment for Alzheimer's disease. Int J Mol Sci; 2014. 15(10):19226-19238. DOI: 10.3390/ijms151019226

10. Upadhyay G, Shankar S, Srivastava RK. Stem cells in neurological disorders: Emerging therapy with stunning hopes. Mol Neurobiol; 2015. 52(1):610-625. DOI: $10.1007 / \mathrm{s} 12035-014-8883-6$

11. Levy YS, Stroomza M, Melamed E, Offen D. Embryonic and adult stem cells as a source for cell therapy in Parkinson's disease. J Mol Neurosci MN; 2004. 24(3):353-386. DOI: 10.1385/JMN:24:3:353

12. Beers DR, Henkel JS, Zhao W, Wang J, Huang A, Wen $\mathrm{S}$, et al. Endogenous regulatory $\mathrm{T}$ lymphocytes ameliorate amyotrophic lateral sclerosis in mice and correlate with disease progression in patients with amyotrophic lateral sclerosis. Brain J Neurol; 2011. 134(Pt 5):1293-1314. DOI: 10.1093/brain/awr074

13. Ruiz Castaneda M. Surface fixation: A new method of detecting certain immunological reactions. Proc Soc Exp Biol Med; 1950. 73(1):46-49.

14. Ruiz Castaneda M. Surface fixation as a practical method for diagnosis of brucellosis in man and animals. Proc Soc Exp Biol Med; 1953. 83(1):36-39. DOI: $10.3181 / 00379727-83-20257$

15. Njoku-Obi AN. Serologic aspects of listeriosis: the antigen-fixation test. In: Symposium on listeric infection. 2nd ed. Montana State College, Bozeman; 1963. p. 223-226.

16. Ruíz Castañeda M. A simple test for detection of specific and unspecific immunological reactions in cancer. Arch Invest Med (Mex); 1980. 11(1):83-93. PMID: 7396635

17. Instituto de Alergias y Autoinmunidad Dr. Maximiliano Ruíz Castañeda A.C. Available from: http://www.iaamrc.com. Accessed November 2, 2020.

18. Ruiz-Castañeda M, Gutiérrez de Velásco-Hernández C, Gómez-Ortiz ME, Pillado-Hernández MT. [Antigenic treatment of patients with Down's syndrome]. Bol Med Hosp Infant Mex; 1982. 39(12):778-780. PMID: 6220726

19. Ruiz-Castañeda M. [Urodiagnosis]. Bol Med Hosp Infant Mex; 1982. 39(12):781-783. PMID: 6220727

20. Oskarsson B, Gendron TF, Staff NP. Amyotrophic Lateral Sclerosis: An Update for 2018. Mayo Clin Proc; 2018. 93(11):1617-1628.

DOI: 10.1016/j.mayocp.2018.04.007

21. Tyndall AJ, Joly F, Carbonne B, Deligny CN, Farge DC. Pregnancy and childbirth after treatment with autologous hematopoietic stem cell transplantation for severe systemic sclerosis requiring parenteral nutrition. Ethical issues. Clin Exp Rheumatol; 2008. 26(6):1122-1124. PMID: 19210885

22. Alexander T, Arnold R, Hiepe F, Radbruch A. Resetting the immune system with immunoablation and autologous haematopoietic stem cell transplantation in autoimmune diseases. Clin Exp Rheumatol; 2016. 34(4 Suppl 98):53-57.

PMID: 27586805

23. Obradović D, Tukić L, Radovinović-Tasić S, Petrović B, Elez M, Ostojić G, et al. Autologous hematopoietic stem cell transplantation in combination with immunoablative protocol in secondary progressive multiple sclerosis -A 10-year follow-up of the first transplanted patient. Vojnosanit Pregl; 2016. 73(5):504-508. DOI: 10.2298/VSP150304045O

24. Urushitani M, Ezzi SA, Julien J-P. Therapeutic effects of immunization with mutant superoxide dismutase in 
mice models of amyotrophic lateral sclerosis. Proc Natl Acad Sci U S A; 2007. 104(7):2495-2500.

DOI: $10.1073 /$ pnas.0606201104

25. Caballero-Hernández D, Toscano MG, CejudoGuillen M, García-Martin ML, López S, Franco JM, et al. The «Omics» of Amyotrophic Lateral Sclerosis. Trends Mol Med; 2016. 22(1):53-67. DOI: $10.1016 /$ j.molmed.2015.11.001

26. Liebau S, Vaida B, Storch A, Boeckers TM. Maturation of synaptic contacts in differentiating neural stem cells. Stem Cells; 2007. 25(7):1720-1729. DOI: $10.1634 /$ stemcells.2006-0823

27. Li J, Lepski G. Cell transplantation for spinal cord injury: a systematic review. BioMed Res Int; 2013. 2013:786475. DOI: 10.1155/2013/786475

28. Riley J, Federici T, Polak M, Kelly C, Glass J, Raore $\mathrm{B}$, et al. Intraspinal stem cell transplantation in amyotrophic lateral sclerosis: A phase I safety trial, technical note, and lumbar safety outcomes. Neurosurgery; 2012. 71(2):405-416. DOI: 10.1227/NEU.0b013e31825ca05f

29. Xu L, Yan J, Chen D, Welsh AM, Hazel T, Johe K, et al. Human neural stem cell grafts ameliorate motor neuron disease in SOD-1 transgenic rats. Transplantation; 2006. 82(7):865-875. DOI: 10.1097/01.tp.0000235532.00920.7a

30. Guo X, Johe K, Molnar P, Davis H, Hickman J. Characterization of a human fetal spinal cord stem cell line, NSI-566RSC, and its induction to functional motoneurons. J Tissue Eng Regen Med; 2010. 4(3):181-193. DOI: $10.1002 /$ term.223

31. Borlongan CV, Evans A, Yu G, Hess DC. Limitations of intravenous human bone marrow CD133+ cell grafts in stroke rats. Brain Res; 2005. 1048(1-2):116122. DOI: $10.1016 /$ j.brainres.2005.04.087

32. Martínez HR, González-Garza MT, Moreno-Cuevas JE, Caro E, Gutiérrez-Jiménez E, Segura JJ. Stem-cell transplantation into the frontal motor cortex in amyotrophic lateral sclerosis patients. Cytotherapy; 2009. 11(1):26-34.
DOI: 10.1080/14653240802644651

33. Batista CE, Mariano ED, Marie SKN, Teixeira MJ, Morgalla M, Tatagiba $M$, et al. Stem cells in neurology--current perspectives. Arq Neuropsiquiatr; 2014. 72(6):457-465. DOI: 10.1590/0004$282 \times 20140045$.

34. Glass JD, Boulis NM, Johe K, Rutkove SB, Federici $\mathrm{T}$, Polak M, et al. Lumbar intraspinal injection of neural stem cells in patients with amyotrophic lateral sclerosis: Results of a phase I trial in 12 patients. Stem Cells; 2012. 30(6):1144-1151.

DOI: $10.1002 /$ stem. 1079

35. Mayaux J, Lambert J, Morélot-Panzini C, GonzalezBermejo J, Delemazure J, Llontop C, et al. Survival of amyotrophic lateral sclerosis patients after admission to the intensive care unit for acute respiratory failure: an observational cohort study. J Crit Care; 2019. 50:54-58. DOI: 10.1016/j.jcrc.2018.11.007

36. Vianello A, Arcaro G, Palmieri A, Ermani M, Braccioni F, Gallan F, et al. Survival and quality of life after tracheostomy for acute respiratory failure in patients with amyotrophic lateral sclerosis. J Crit Care; 2011. 26(3):329.e7-14.

DOI: $10.1016 /$ j.jcrc.2010.06.003

37. Spataro R, Bono V, Marchese S, La Bella V. Tracheostomy mechanical ventilation in patients with amyotrophic lateral sclerosis: Clinical features and survival analysis. J Neurol Sci; 2012. 323(1-2):66-70. DOI: 10.1016/j.jns.2012.08.011

38. Ulhaq Z. Aromatase and neuroregeneration. MNJ (Malang Neurology Journal); 2019. 5(1):48-50. DOI: 10.21776/ub.mnj.2019.005.01.9

39. Antunes J, Karvat J, Bernardino G, Kakihata C, Brancalhão R, Ribeiro L, et al. Histological analysis of wistar rats sciatic nerves subjected to compression and treated with low level laser therapy and neural mobilization. MNJ (Malang Neurology Journal); 2019. 5(2):55-60.

DOI: 10.21776/ub.mnj.2019.005.02.1 